\title{
Using 360-degree videos for virtual reality exposure in CBT for panic disorder with agoraphobia: a feasibility study
}

\author{
Johan Lundin ${ }^{1,4 \star}$ (10), Anders Lundström², Jan Gulliksen ${ }^{2}$, Joakim Blendulf ${ }^{3}$, Kersti Ejeby ${ }^{4}$, \\ Hedda Nyman ${ }^{4}$, Daniel Björkander ${ }^{4}$ and Erik Hedman-Lagerlöf ${ }^{4,5}$ \\ ${ }^{1}$ Centre for Psychiatry Research, Department of Clinical Neuroscience, Karolinska Institutet, Stockholm, Sweden, ${ }^{2}$ KTH Royal \\ Institute of Technology, School of Electrical Engineering and Computer Science, Media Technology and Interaction Design, \\ Stockholm, Sweden, ${ }^{3}$ Film Stockholm, Culture Administration, Region Stockholm, Sweden, ${ }^{4}$ Gustavsberg Primary Care \\ Clinic, Gustavsberg, Sweden and ${ }^{5}$ Section for Pychology \& Osher Center for Integrative Medicine, Department of Clinical \\ Neuroscience, Karolinska Institutet, Stockholm, Sweden \\ ${ }^{\star}$ Corresponding author. Email: johan.lundin.1@ki.se
}

(Received 16 November 2020; revised 3 September 2021; accepted 4 October 2021; first published online 18 November 2021)

\begin{abstract}
Background: Cognitive behavioural therapy (CBT) is an effective treatment for panic disorder with agoraphobia (PDA). However, implementation of some of the procedures involved, particularly in vivo exposure, can be time consuming and taxing for routine health care services. CBT with exposure taking place in virtual reality (VR-CBT) is a more time-efficient option and has shown promising results in the treatment of PDA. However, VR-CBT requires expensive equipment and appropriate virtual environments, which historically has been costly and cumbersome to produce. Thus, access to VR-CBT has been sparse in regular care environments.

Aims: The aim of this study was to investigate whether VR-CBT using filmed virtual environments produced with a low-cost 360-degree film camera can be a feasible and acceptable treatment for PDA when implemented in a primary care context.

Method: This was an open feasibility trial with a within-group design, with assessments conducted at pre-test, post-test, and 6-month follow-up. Participants $(n=12)$ received a 10-12 week treatment programme of VR-CBT and PDA-related symptoms were assessed by the primary outcome measure The Mobility Inventory for Agoraphobia (MIA) and the Panic-Disorder Severity Scale-Self Rated (PDSS-SR).

Results: The results showed that treatment satisfaction was high and participants were significantly improved on PDA-related measures at post-treatment and at 6-month follow-up with large effect sizes (Cohen's $d$ range $=1.46-2.82$ ). All 12 participants completed the treatment.

Conclusions: These findings suggest that VR-CBT with 360-degree video virtual environments delivered to primary care patients with PDA is feasible, acceptable, and potentially efficacious.
\end{abstract}

Keywords: agoraphobia; anxiety disorders; cognitive behaviour therapy; panic disorder; primary care; virtual reality

\section{Introduction}

Panic disorder with agoraphobia (PDA) is characterised by fear of having panic attacks or panicrelated symptoms in situations from where escape is perceived difficult (Pompoli et al., 2016). PDA can be a highly disabling condition for afflicted individuals that, without proper treatment, often follows a chronic course (Kessler et al., 2006). Moreover, PDA is associated

(C) The Author(s), 2021. Published by Cambridge University Press on behalf of the British Association for Behavioural and Cognitive Psychotherapies. This is an Open Access article, distributed under the terms of the Creative Commons Attribution licence (https:// creativecommons.org/licenses/by/4.0/), which permits unrestricted re-use, distribution, and reproduction in any medium, provided the original work is properly cited. 
with extensive economic costs (Roy-Byrne et al., 1999) and high pharmacological utilisation (Deacon et al., 2008).

There is strong empirical support for cognitive behaviour therapy (CBT) in the treatment of PDA (Sánchez-Meca et al., 2010). However, despite demonstrable effectiveness of CBT, studies have shown that only a minority of patients with PDA receive evidence-based treatment (Wolitzky-Taylor et al., 2015), particularly in primary care settings (Marcks et al., 2009). Indeed, one study showed that only a quarter of primary care patients with PDA had recovered three years after intake (Francis et al., 2007).

Evidence-based CBT approaches for PDA emphasise, to varying degrees, catastrophic misinterpretations of bodily symptoms as well as agoraphobic avoidance as key maintenance factors (Clark, 1986; Craske and Barlow, 2014). A key intervention in CBT to target these maintenance factors is exposure. CBT models for PDA diverge slightly in their conceptualisations of why exposure is effective. Traditional exposure models emphasise fear extinction via habituation (Öst, 2008). Cognitive models apply exposure as a pathway to disconfirm fear appraisals regarding anxiety symptom and agoraphobic situations (Clark, 1986; Clark and Salkovskis, 2009). The inhibitory learning model (ILM; Craske, 2014) posits that exposure does not, as once assumed, remove a fear association between a conditioned stimulus (CS) and an unconditioned stimulus (US). Instead, exposure leads to new safe associations, i.e. that the CS does not predict the US. Hence, exposure is effective if conducted in ways that augment and generalise these new safe associations, as they then can function to inhibit the original fear (Craske, 2014). While these approaches digress somewhat in their theoretical underpinnings, the two latter approaches overlap in their view that exposure is most effective when it facilitates corrective learning regarding panic symptoms and their consequences in agoraphobic situations (Craske, 2014).

Although exposure is a highly effective intervention for PDA (Öst, 2008; Sánchez-Meca et al., 2010) it can be difficult to implement practically. First, patients need to enter the specific situations they fear, accompanied by the clinician. This is a time-consuming exercise involving travelling to and from the clinic and finding the appropriate locations. Second, the clinician and patient must have access to the 'right environments', which can be difficult depending on the patient's specific fears as well as where the clinic is located. These are particularly challenging obstacles in primary care where clinicians are pressed for time and sessions are usually restricted to $45-50$ minutes per patient. Third, the nature of exposure, which is to face the stimuli one fears, may for some people be perceived as too aversive leading to treatment drop-outs or avoidance of treatment-seeking (Botella et al., 2007; Malbos et al., 2013).

Given these predicaments, it is critical to develop alternative forms of exposure-based interventions that will be equally effective, acceptable to patients, and less time-consuming. In the last decades numerous studies have demonstrated that exposure-based CBT via virtual reality (VR-CBT) is effective in the treatment of anxiety disorders, particularly for specific phobias (Carl et al., 2019; Powers and Emmelkamp, 2008). VR technology is used to display computer-generated virtual environments (VE), analogous to the real world, via a VR headset, which allows the subject to experience the environment in 360 degrees and/or 3D. This technology immerses the user in the VE, making him or her feel as if the experience is real (Botella et al., 2017). VR-CBT is often referred to as virtual reality exposure therapy (VRET) in the literature. This article will use to the term VR-CBT when referring to treatment protocols that combine VR-exposure with other CBT techniques, e.g. cognitive restructuring and VRET when referring to VR-exposure only.

A number of possible advantages of VR-CBT and VRET compared with standard face-to-face CBT have been proposed: therapy sessions are less time-consuming, the clinician has more control of the environment, one can repeat and/or prolong exposures more easily, it is possible to vary between phobic stimuli quickly and easily and it may be more acceptable by patients (Emmelkamp, 2005; Garcia-Palacios et al., 2007). 
A few studies have examined the effectiveness of VR-CBT and VRET for PDA (Carl et al., 2019; Opriş et al., 2012). Two randomised controlled trials (RCT) investigated VRET compared with in vivo exposure therapy. Both studies found VRET to be as efficacious as in vivo exposure and superior to wait-list (Botella et al., 2007; Pelissolo et al., 2012). One RCT compared VR-CBT with standard CBT. This study showed a slight superiority for in vivo exposure on measures of panic symptoms but no difference for agoraphobic avoidance (Meyerbroeker et al., 2013).

Overall, these studies yield promising results but there are limitations. To our knowledge no previous study has investigated effectiveness of VR-CBT for PDA in a routine care setting. Thus, little is known whether VR-exposure is feasible and acceptable in routine health care. Furthermore, a possible drawback with the above studies in terms of clinical utility is that they utilised computer animated VEs. Producing these VEs has been reported to be a costly and laborious procedure, which may reduce cost-effectiveness and the overall clinical utility of VR-CBT (Meyerbroeker et al., 2013). While recent technological advances have significantly eased the process of developing computer animated VEs (Lindner et al., 2017), there may be other pathways to simplify the procedure of producing clinically beneficial VEs.

An alternative to computer animated VEs is to use film cameras and filmed locations and scenarios as VEs. Given the low costs of 360-degree film cameras and the user-friendly procedures for filming and editing, this method allows for a flexible use of VR-CBT as VEs can be constantly added in according to patient needs. Furthermore, the photorealism of filmed VEs may augment the experiential qualities of the VR-experience.

To our knowledge, following an extensive review of the research literature, there is a growing body of research on VR applications using 360-degree films in somatic medical settings, e.g. anaesthetic care (Sullivan et al., 2018), but no previous VR studies have developed and utilised filmed VEs in the treatment of PDA.

The aim of this feasibility study was to investigate if VR-CBT using filmed VEs can be feasible and acceptable to patients with PDA when implemented in a primary care setting. We also investigated preliminary efficacy by exploring pre- to post-changes in panic attacks, agoraphobic avoidance, depressive symptoms, daily functioning and quality of life. We expected that patients would be satisfied with the treatment and that adverse events would be rare.

\section{Method}

\section{Design}

This was an open feasibility trial of VR-CBT over $10-12$ weeks for adults with PDA $(n=12)$ using a pre-test-post-test design. The trial was conducted at Gustavsberg's Primary Care Clinic in Stockholm, Sweden, and assessments were carried out at baseline, after treatment and at 6-months follow-up (6MFU).

\section{Participants and recruitment}

Participants were recruited from the regular flow of patients at the primary care clinic, or via selfreferral. Information about the study was published on the clinic's official website and advertised in a newspaper with regional coverage.

To be included in the study, participants had to: (a) have agoraphobia with concurrent or previous panic disorder according to DSM-5 diagnostic criteria (American Psychiatric Association, 2013) as their principal diagnosis, (b) agree not to undergo any other psychological treatment during the study, (c) be at least 18 years old, (d) have basic reading and writing skills in Swedish, (e) not have serious concurrent psychiatric problems such as substance abuse, psychosis, bipolar disorder or severe depression, (f) if on prescribed 
Table 1. Description of the participants

\begin{tabular}{lll}
\hline & & VR-CBT $(n=12)$ \\
\hline \multirow{2}{*}{ Gender } & Women & $9(75 \%)$ \\
& Men & $3(25 \%)$ \\
& Mean age $(S D)$ & $40.8(15 \%)$ \\
Occupational status & Min-max & $23-62$ \\
& Working full-time/part-time & $6(50 \%)$ \\
& Student & $1(8 \%)$ \\
& Unemployed & $3(25 \%)$ \\
Marital and parental status & Sick leave & $1(8 \%)$ \\
Previous CBT for PDA & Parental leave & $1(8 \%)$ \\
Co-morbid conditions & Married or de facto & $8(67 \%)$ \\
& Parent, yes & $8(67 \%)$ \\
& Yes & $5(42 \%)$ \\
& No & $7(58 \%)$ \\
& Generalized anxiety disorder & $1(8 \%)$ \\
& Obsessive compulsive disorder & $1(8 \%)$ \\
& Depression & $3(25 \%)$ \\
& Social anxiety disorder & $1(8 \%)$ \\
\end{tabular}

VR-CBT, virtual reality cognitive behaviour therapy; PDA, panic disorder with agoraphobia. Exhaustion disorder is a stress-related disorder that only exists in the Swedish diagnostic system.

medication for anxiety, be on stable dosage for a minimum of 4 weeks preceding treatment, and (g) not having had CBT for PDA in the last year. Psychiatric diagnoses other than those mentioned above were accepted as co-morbid conditions provided that they were assessed as being secondary to PDA.

Referred patients who were interested in participating had a face-to-face session with a licensed psychologist at the clinic who provided detailed information about the study and conducted a diagnostic assessment interview including the Mini International Neuropsychiatric Interview (MINI) (Sheehan et al., 1998).

Note that although agoraphobia was the only required diagnosis, all participants also met diagnostic criteria for panic disorder (PD). Therefore, we describe this study as an investigation of VR-CBT for patients with PDA. Table 1 shows demographic and clinical characteristics of the participants.

\section{Outcome measures}

Two outcome measures were used to assess effectiveness of VR-CBT for PDA: the primary measure Mobility Inventory for Agoraphobia (MIA; Chambless et al., 1985) and the Panic Disorder Severity Scale-Self Rated (PDSS-SR; Houck et al., 2002). The MIA is a 51-item selfreport scale measuring severity of agoraphobic avoidance divided into two subscales, when accompanied and when alone (scores are computed by calculating the average for each subscale and range from 1 to 5). The MIA measures avoidance on a 5-point Likert scale with descriptors: $1=$ never avoid, $2=$ rarely avoid, $3=$ avoiding half the time, $4=$ avoiding most of the time, and $5=$ always avoid. Good psychometric properties have been demonstrated for the MIA with high internal consistency (Cronbach's $\alpha=0.95)$ (Chambless et al., 2011) and good test-re-test reliability $(r=.89)$.

The PDSS-SR is a 7-item self-report instrument (scale range from 0 to 28 ) that measures panic disorder severity. The items are rated on a 5-point scale and cover central features of panic disorder: frequency of attacks, distress during panic, panic-focused anticipatory anxiety, agoraphobic avoidance of situations, avoidance of physical symptoms, and impairment in 
work functioning and social functioning. The PDSS-SR has demonstrated high internal consistency (Cronbach's $\alpha=0.92$ ) as well as good test-re-test reliability $(r=0.81)$.

Secondary outcome measures included were the Patient Health Questionnaire-9 (PHQ9) (Kroenke et al., 2001) to assess depression, the World Health Organization Disability Assessment (WHODAS) (Ustün et al., 2010) to assess functional impairment and World Health Organization Quality of Life questionnaire (WHOQOL) (The WHOQOL Group, 1995) to assess quality of life.

To assess acceptability, the Client Satisfaction Questionnaire-8 (CSQ-8) was administered after treatment (Attkinson and Zwick, 1982). The CSQ-8 is an 8-item scale (scale range 8 to 32) and entails items that cover different facets of treatment satisfaction, e.g. 'To what extent has our program met your needs?' and 'Did you get the kind of service you wanted?'. The CSQ-8 has demonstrated good psychometric properties including high reliability and construct validity (Attkisson and Zwick, 1982; De Brey, 1983). All self-report questionnaires were administered online.

\section{Treatment}

This treatment protocol consisted of 10-12 weekly 60-minute sessions (mean number of sessions $=11.5 ; S D=0.76$ ) and was largely based on Clark and Salkovskis' (2009) cognitive behavioural model, one of the most empirically supported psychosocial treatments for PDA. In addition, selected principles from the ILM were adopted for this treatment (Craske and Simos, 2013). ILM proposes specific treatment strategies aimed at augmenting and optimising exposure exercises. ILM principles applied in this treatment were violation expectancy, deepened extinction and variability of conditioned stimuli.

Treatment sessions were conducted by three licensed psychologists with experience in CBT for PDA who worked at the clinic. Patients were seen weekly. Supervision was conducted by a senior psychologist with extensive training in the treatment method who was one of the therapists in the trial.

The following components were included in the treatment: developing an individual PDAmaintenance model, psychoeducation, verbal cognitive restructuring and VR exposure. Most sessions were devoted to exposure through VR. The VEs used in a given treatment were selected in collaboration with the patient. Typically, those that simulated or resembled situations that the patient found anxiety-provoking in real life were chosen. Exposures were set up as behavioural experiments, targeting disconfirmation of feared expectancies by remaining in the $\mathrm{VE}$ despite anxiety and without performance of safety behaviours. On occasion, in order to increase anxiety-invoking stimuli, feared bodily symptoms were induced prior to VR exposures through additional exercises, for instance hyperventilating before putting on the VR-headset and entering the VE. In addition, auditory stimuli reminiscent of panic symptoms, e.g. sounds of a racing heartbeat, were induced during exposure via headphones connected to the VR-headset. Exposure was followed by discussion and consolidation of learning obtained from the exercise. Homework exercises consisted of filling out weekly panic diaries, filling out individual PDA models and answering written questions regarding what they had learned in the previous session. Patients were not explicitly given exposure tasks for homework as the main aim was to test VR exposure. However, they were encouraged to utilise what they learned in therapy in their day-to-day life. For a detailed description of treatment content, see Table 2.

\section{Apparatus and VE environments}

The apparatus and VEs (360-degree films) were developed collaboratively with psychologists, clinical researchers, human computer interaction (HCI) researchers, and filmmakers. The 
Table 2. Treatment structure

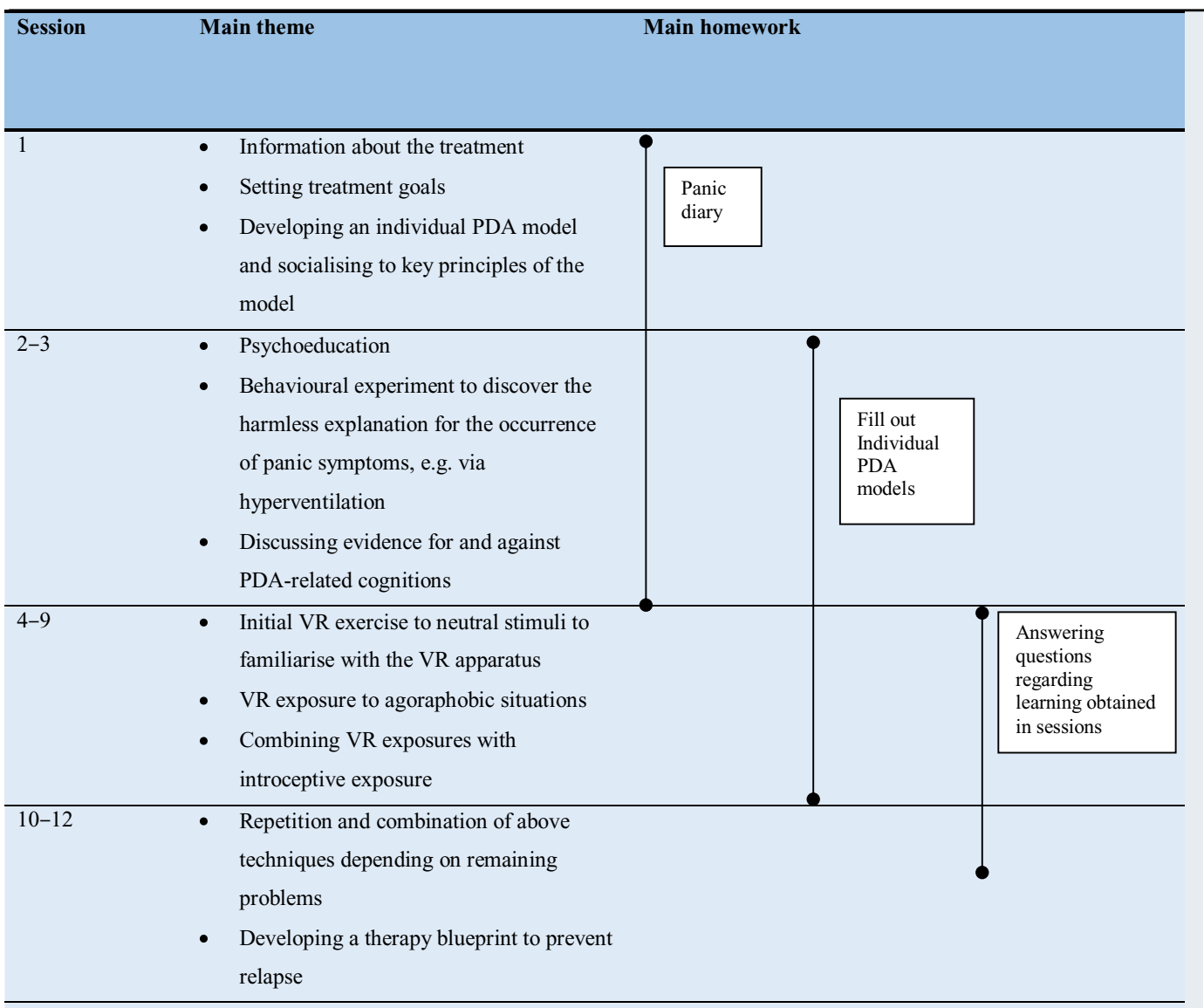

PDA, panic disorder with agoraphobia; VR, virtual reality.

process was led by an HCI researcher and conducted through a series of workshops focusing on determining needs and requirements for VR-CBT in the treatment of PDA. These workshops covered design of the apparatus and deciding suitable treatment scenarios.

The VEs selected were typical situations feared and avoided by individuals with PDA. We filmed inside a subway carriage, a walking tunnel, a busy train station, an elevator, an auditorium, and a tall bridge. Permission to film in the subway was given by the public transport authorities. As 360-degree films taken in motion are known to cause motion sickness, each VE was constructed through a series of stationary 360-degree films at different locations within what we refer to as scenarios. For example, one subway scenario consisted of seven consecutive films: (1) outside the subway, (2) the ticket gates, (3) on the subway platform, (4) inside the train, (5) on the destination platform, (6) at the exit, and (7) outside the station. In total we filmed six situations containing between three and seven scenarios.

All the VEs were captured using a Samsung VR 360 camera. The equipment used for the VR exposure consisted of a powerful gaming computer and an HTC Vive head-mounted display (HMD) (see: www.vive.com). See Fig. 1 for an overview of the set-up. The psychologists were 


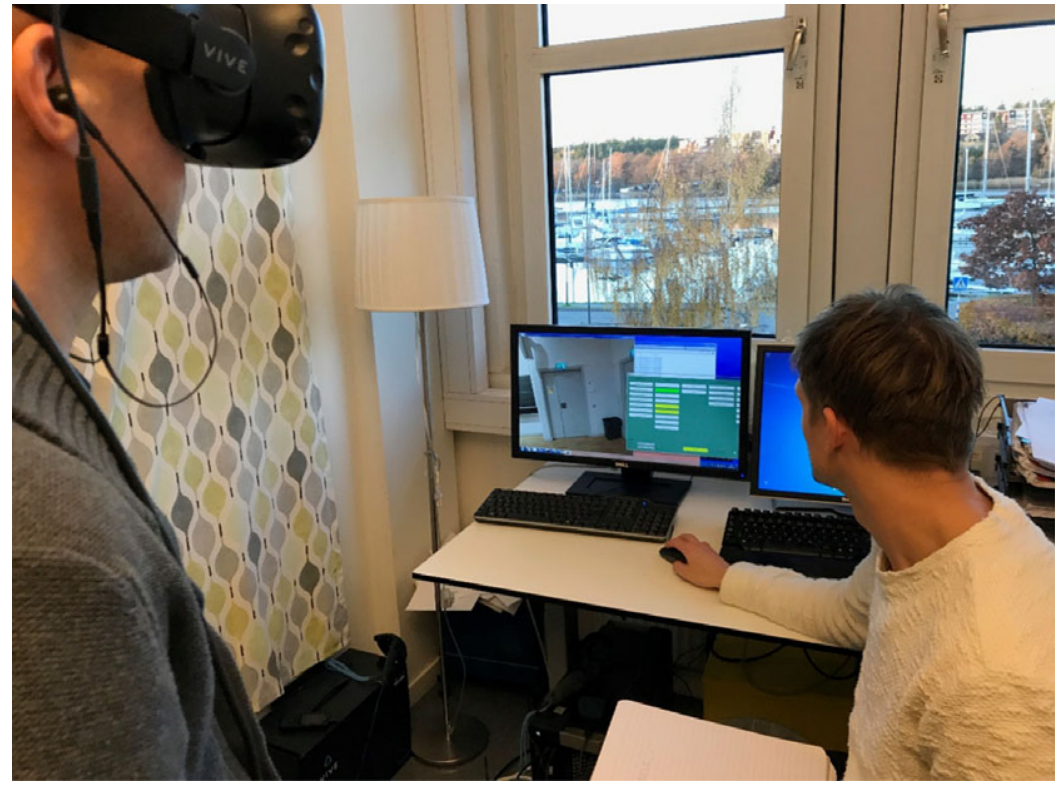

Figure 1. Photo of a typical VR exposure session at the clinic. The picture shows the VR headset worn by the patient (left) and how the therapist (right) uses the controller to adapt the VEs to the patient's needs.

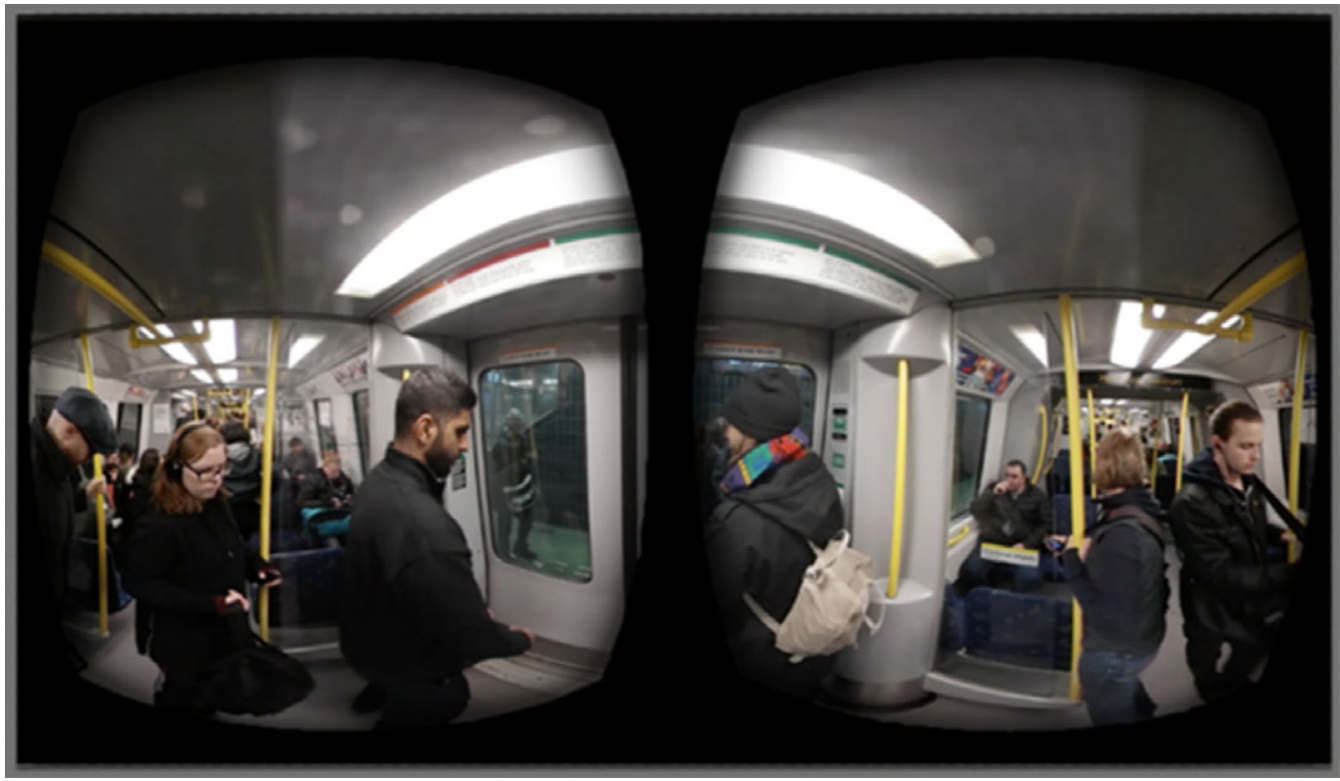

Figure 2. Image of a VE from the patient perspective when wearing the VR headset.

able to select and control VEs, adjust sound levels, add stressors and view what the patient was seeing in the HMD, on their computer via a video player developed for the study. For an image of a VE from the patient perspective, see Fig. 2. A detailed description of the apparatus and technical set-up is provided in the Supplementary material. 
Table 3. Means and effect sizes on all outcome measures from baseline to post-treatment and 6-month follow-up

\begin{tabular}{|c|c|c|c|c|c|c|c|}
\hline \multirow[b]{2}{*}{ Measure } & \multirow[b]{2}{*}{$\begin{array}{c}\text { Pre } \\
\text { Mean (SD) }\end{array}$} & \multirow[b]{2}{*}{$\begin{array}{c}\text { Post } \\
\text { Mean }(S D)\end{array}$} & \multirow[b]{2}{*}{$\begin{array}{c}\text { 6MFU } \\
\text { Mean (SD) }\end{array}$} & \multicolumn{2}{|c|}{ Pre-Post } & \multicolumn{2}{|c|}{ Pre-6MFU } \\
\hline & & & & $p$-value & $\begin{array}{l}\text { Cohen's } d \\
(95 \% \mathrm{Cl})\end{array}$ & $p$-value & $\begin{array}{l}\text { Cohen's } d \\
(95 \% \mathrm{Cl})\end{array}$ \\
\hline MIA (alone) & $3.15(0.6)$ & $2.02(0.8)$ & $1.66(0.44)$ & $<.001$ & $1.46(0.82-2.11)$ & $<.001$ & $2.82(1.07-4.67)$ \\
\hline MIA (accom.) & $2.49(0.5)$ & $1.4(0.47)$ & $1.37(0.4)$ & $<.001$ & $2.09(1.02-3.17)$ & $<.001$ & $2.25(1.14-3.37)$ \\
\hline PDSS-SR & $10.5(4.7)$ & $3(2.9)$ & $1.9(3.4)$ & $<.001$ & $1.91(0.57-3.25)$ & $<.001$ & $2.06(0.78-3.34)$ \\
\hline PHQ-9 & $7.67(5.3)$ & $2(2.0)$ & $1.5(2.6)$ & $<.001$ & $1.19(0.41-1.96)$ & .008 & $1.40(0.26-2.54)$ \\
\hline WHODAS & $24.5(18.8)$ & $9.03(8.8)$ & $11.25(15.9)$ & .003 & $0.85(0.26-1.41)$ & .035 & $0.75(0.01-1.48)$ \\
\hline WHOQOL & $81.94(9.3)$ & $88.89(10.0)$ & $91.67(12.0)$ & .016 & $0.72(0.09-1.34)$ & .018 & $0.87(0.25-1.48)$ \\
\hline CSQ & & $30.1(2.71)$ & & & & & \\
\hline
\end{tabular}

Pre, pre-treatment; Post, post-treatment; 6MFU, six-month follow-up; MIA, Mobility Inventory; PDSS-SR, Panic Disorder Severity Scale-self rated; PHQ-9, Patient Health Questionnaire; WHODAS, World Health Organisation Disability Assessment; WHOQOL, World Health Organisation Quality of Life; CSQ, Client Satisfaction Questionnaire.

\section{Statistical analysis}

Statistical analyses were conducted using SPSS 22.0 (IBM, Chicago, USA) based on the intention to treat principle, i.e. all study participants who completed pre-treatment assessment were included in the analyses regardless of level of adherence to the treatment. We used paired $t$-tests to investigate change over time and calculated effect sizes (Cohen's $d$ ) based on pooled standard deviations. As a dichotomous measure of treatment response for the PDA-related measures MIA and PDSS-SR we used previously established definitions (Furukawa et al., 2009; Ruwaard et al., 2010; NHS England, 2018). In line with most investigators, we only included the MIA avoidance alone subscale in the analysis, as it has stronger construct validity than the accompany subscale (Chambless et al., 2011). This meant that an improvement of at least 0.73 points on the MIA-alone scale and 5 points on the PDSS-SR was required for a participant to be defined as a responder on the respective measures. Calculations showed that with an expected within-group effect of Cohen's $d=0.80$ on the primary outcomes, 14 participants were required to yield $80 \%$ power, given an alpha level of .05 .

\section{Results}

\section{Attrition}

There were no missing data on primary and secondary outcome measures at baseline or post-treatment. At 6MFU, 10 participants of $12(83 \%)$ provided data.

\section{Treatment satisfaction and adverse events}

All patients completed the treatment. The mean score on the CSQ (scale range 8-32) was 30.1 $(S D=2.71)$, indicating high overall treatment satisfaction and acceptability of the VR-CBT format. No serious adverse events were reported. One participant reported that the treatment initially increased panic attack frequency but that it declined during treatment and had no negative effects on overall wellbeing. Another participant reported an increase in work-related stress, which had a mild negative effect on overall wellbeing.

\section{Change in PDA symptoms}

Table 3 presents means, SDs and effect sizes for all outcome measures at baseline, post-treatment and $6 \mathrm{MFU}$. There was a significant baseline to post-treatment reduction of agoraphobic avoidance as measured with the MIA both when alone $\left(t_{11}=7.18, p=0.001\right)$ and when accompanied 
$\left(t_{11}=7.67, p=0.001\right)$ with large effect sizes for both subscales. There was also a significant baseline to post-treatment reduction of panic disorder severity as measured by the PDSS-SR $\left(t_{11}=5.25, p=0.001\right)$ with a large effect size. At 6MFU, agoraphobic avoidance remained significantly reduced compared with baseline according to the MIA when alone $\left(t_{9}=8.62\right.$, $p=0.001)$ and when accompanied $\left(t_{9}=7.61, p=0.001\right)$. Reduction in panic disorder severity was also significant from baseline to $6 \mathrm{MFU}\left(t_{9}=5.84, p=0.001\right)$.

\section{Responder rates}

On the MIA-alone scale, nine patients (75\%) were treatment responders post-treatment. At 6MFU, eight of the 10 patients (80\%) who completed the assessment were treatment responders. On the PDSS-SR, nine patients (75\%) were treatment responders at posttreatment. Of the 10 patients who completed $6 \mathrm{MFU}$ assessment, nine patients (90\%) were treatment responders.

\section{Secondary outcomes}

There was a significant baseline to post-treatment reduction of depressive symptoms, as measured by the PHQ-9 $\left(t_{11}=4.4, p=0.001\right)$ with a large effect size. There was also a significant improvement baseline to post-treatment on measures of functional impairment and quality of life: WHODAS $\left(t_{11}=3.68, p=0.005\right)$ and WHOQOL $\left(t_{11}=-2.83, p=0.04\right)$ with large and moderate effect sizes, respectively. There were also significant improvements in scores on the PHQ-9 $\left(t_{9}=3.32, p=0.01\right)$ and WHOQOL $\left(t_{9}=-2.88, p=0.02\right)$ from baseline to $6 \mathrm{MFU}$. There was a significant improvement with regard to overall functional impairment from baseline to $6 \mathrm{MFU}$ as measured by the WHODAS $\left(t_{9}=2.47, p=0.04\right)$ with a moderate effect size.

\section{Discussion}

The aim of this study was to investigate whether VR-CBT for PDA using filmed VEs was feasible, acceptable and potentially efficacious when implemented in a primary care setting.

Patients were highly satisfied with the treatment, as indicated by the high mean score on the CSQ, and adverse events were rare. Also, none of the patients dropped out, which indicates that the treatment was acceptable. As a comparison, a meta-analysis of therapeutic studies on PD found mean drop-out rates of $15.1 \%$ for CBT trials (Mitte, 2005). This gives tentative support for the hypothesis that VR exposure may be perceived less aversive than in vivo exposure, and it may be that VR-CBT has the potential to reach patients who would hesitate to go through conventional CBT treatments involving exposure therapy.

The within-group effect sizes were large on PDA-related outcome measures MIA and PDSS-SR from baseline to post-treatment and from baseline to $6 \mathrm{MFU}$. At post-treatment, $75 \%$ were classified as responders on the PDSS-R as well as on the MIA-alone scale. At $6 \mathrm{MFU}, 80 \%$ were classified as responders on the PDSS-R and $90 \%$ on the MIA-alone. There was also a substantial improvement in terms of symptoms of depression, quality of life and daily functioning. Worth noting is that the mean score at baseline on the PHQ-9 was 7.67, which is under the remission cut-off score of 9 . This indicates that depression severity was rather mild for this group prior to treatment.

Altogether, these findings provide preliminary support for VR-CBT as a feasible treatment for patients with PDA when conducted in a primary care setting. The results on the outcome measures are promising, but replication in a larger controlled trial is needed. The effects seen in this study are similar to those reported in meta-analytic reviews investigating face-to-face CBT for PDA (Norton and Price, 2007; Sánchez-Meca et al., 2010). 
So, what are the potential clinical implications of this study? We believe that VR-CBT in this format has the potential to increase accessibility to exposure-based treatment in routine care due to a number of factors. Considering that all exposure took place at the clinic through VR within a maximum session time of 60-minutes, there are good reasons to assume that VR-CBT (1) is more time-efficient than in vivo exposure, (2) eases accessibility to relevant situational stimuli which facilitates exposure-based treatment, and (3) can facilitate exposure in remote clinics where access to agoraphobic situations is sparse. Finally, we believe that VR-CBT can be potentially cost-effective in comparison with conventional face-to-face CBT given that no therapist time is spent on exposures outside of the clinic. Of course, this needs to be empirically investigated.

One central aspect of VR-CBT is to what extent it is possible to implement in routine care, where costs and resources required for the health care provider to set up the VR equipment are important factors. In this study we used HTC Vive for the VR set-up, which requires installation of wall-mounted satellites. This may not be practical to implement in most primary care clinics. Importantly, recent advances in VR technology such as the Oculus Quest has made it possible to expose the patient to the same VEs as in the present study without the need for a computer because the headset contains the necessary hardware. Such purely headset-based VR solutions have lowered the equipment cost by a factor of 5 compared with our set-up. Furthermore, these new mobile headsets appear well suited for 360-degree video in clinical practice as they can easily be moved between treatment rooms and potentially also be brought home by the patient for in-between session home exposure.

While we regard the results of the present study as highly promising, they must be viewed in the light of the study's limitations. We used a within-group design without randomisation to a control condition and therefore we cannot draw inferences about whether changes in the outcomes were caused by the treatment. However, research has shown that PDA is chronic in the absence of treatment for the majority of afflicted individuals (Kessler et al., 2006), which makes it unlikely that all effects were attributable to spontaneous improvements. Randomised controlled trials are nonetheless needed to establish the treatment's efficacy.

Another limitation is that, as VR exposure was combined with other interventions it cannot be concluded that VR exposure was a 'necessary ingredient', responsible for treatment effects. Furthermore, while the treatment did not include planned exposure exercises as homework, patients were encouraged to utilise what they learned in therapy in their daily life. It was not controlled to what extent (or whether at all) participants engaged in exposures between sessions. As such it may be that patients engaged in exposure of their own accord and that such endeavours may have contributed to treatment outcomes. If that were the case, we would however interpret that as VR-exposure facilitated in vivo exposure without further effort from the therapist. This is speculative and the intention of this study was not to disentangle the relative contribution of a specific technique but rather to investigate the effect of the VR-CBT treatment package as a whole. However, for future studies, it would be valuable to isolate this intervention more rigorously in order to investigate the specific contribution of VR exposure.

Finally, there is a limitation with regard to the sample in the present study. The mean score for this sample on the PDSS-SR was 10.5 at pre-treatment, which is slightly lower than what is commonly reported in studies on PDA. One large study investigating psychometric properties for the PDSS-SR reported a mean of 12.43 for their PD/PDA sample (Svensson et al., 2019). The generalisability of our results to populations with more severe panic symptoms is thus uncertain. The moderate pre-treatment symptom levels in the present study were partly due to an outlier with a very low score (with this participant removed, the pre-treatment mean was 11.5 on the PDSS-SR).

Despite these limitations, and considering the large effect sizes, high degree of self-reported treatment satisfaction and no drop-outs, we conclude that VR-CBT with 360-degree video 
VEs appears to be a feasible, acceptable and potentially efficacious treatment option for PDA suitable in primary care settings.

Supplementary material. To view supplementary material for this article, please visit https://doi.org/10.1017/S1352 465821000473

\section{Acknowledgements. None.}

Author contributions. Johan Lundin: Conceptualization (equal), Data curation (equal), Formal analysis (equal), Investigation (equal), Methodology (equal), Project administration (Lead), Resources (equal), Supervision (lead), Visualization (supporting), Writing-original draft (lead), Writing-review \& editing (equal); Anders Lundström: Conceptualization (equal), Funding acquisition (equal), Investigation (equal), Methodology (equal), Project administration (equal), Resources (equal), Software (lead), Visualization (equal), Writing-review \& editing (equal); Jan Gulliksen: Conceptualization (supporting), Funding acquisition (equal), Project administration (supporting), Resources (equal), Software (supporting), Writing-review \& editing (supporting); Joakim Blendulf: Conceptualization (equal), Funding acquisition (equal), Project administration (equal), Resources (equal), Software (equal), Visualization (equal), Writingreview \& editing (supporting); Kersti Ejeby: Conceptualization (supporting), Funding acquisition (equal), Methodology (supporting), Project administration (supporting), Resources (equal), Writing-review \& editing (supporting); Hedda Nyman: Conceptualization (supporting), Investigation (equal), Project administration (supporting), Resources (equal), Writing-review \& editing (supporting); Daniel Björkander: Conceptualization (supporting), Investigation (equal), Project administration (supporting), Resources (equal), Writing-review \& editing (supporting); Erik Hedman-Lagerlöf: Conceptualization (equal), Data curation (equal), Formal analysis (equal), Funding acquisition (equal), Investigation (supporting), Methodology (lead), Project administration (equal), Resources (equal), Supervision (lead), Writing-review \& editing (equal).

Financial support. This project was supported by research grants from Region Stockholm (Film Stockholm and Innovationsfonden). The funding organization (Region Stockholm) is a public institution and neither of the funding organizations had any role in the design and conduct of the study; in the collection, management, and analysis of the data; or in the preparation, review and approval of the manuscript

Conflicts of interest. The authors declare none.

Ethics statements. All participants provided written informed consent and the study was approved by the Regional Ethics Review Board in Stockholm (ID: 2016/2552-31/). This study was registered at: Clinicaltrials.gov (ID NCT03101332); https:// clinicaltrials.gov/ct2/show/NCT03101332

Data availability. With respect to the legal framework regulating access to research data in Sweden and the European Union, the data are not freely accessible due to regulations regarding personal integrity in research, public access and privacy; each request for data must therefore be assessed by the Swedish Ethical Review Authority. Data access can be given after this assessment.

\section{References}

American Psychiatric Association (2013). Diagnostic and Statistical Manual of Mental Disorders: DSM-5. Arlington, VA, USA: American Psychiatric Association.

Attkisson, C. C., \& Zwick, R. (1982). The Client Satisfaction Questionnaire. Psychometric properties and correlations with service utilization and psychotherapy outcome. Evaluation and Program Planning, 5, 233-237.

Botella, C., Fernández-Álvarez, J., Guillén, V., García-Palacios, A., \& Baños, R. (2017). Recent progress in virtual reality exposure therapy for phobias: a systematic review. Current Psychiatry Reports, 19, 42. https://doi.org/10.1007/s11920-0170788-4

Botella, C., Garcia-Palacios, A., \& Villa, H. (2007) Virtual reality exposure in the treatment of panic disorder and agoraphobia: a controlled study. Clinical Psychology and Psychotherapy, 14, 164-175.

Carl, E., Stein, A. T., Levihn-Coon, A., Pogue, J. R., Rothbaum, B., Emmelkamp, P., Asmundson, G. J. G., Carlbring, P., \& Powers, M. B. (2019). Virtual reality exposure therapy for anxiety and related disorders: a meta-analysis of randomized controlled trials. Journal of Anxiety Disorders, 61, 27-36. https://doi.org/10.1016/j.janxdis.2018.08.003

Chambless, D. L., Caputo, G. C., Jasin, S. E., Gracely, E. J., \& Williams, C. (1985). The Mobility Inventory for Agoraphobia. Behaviour Research and Therapy, 23, 35-44. https://doi.org/10.1016/0005-7967(85)90140-8

Chambless, D. L., Sharpless, B. A., Rodriguez, D., McCarthy, K. S., Milrod, B. L., Khalsa, S.-R., \& Barber, J. P. (2011). Psychometric properties of the mobility inventory for agoraphobia: convergent, discriminant, and criterion-related validity. Behavior Therapy, 42, 689-699. https://doi.org/10.1016/j.beth.2011.03.001 
Clark, D. (1986). A cognitive approach to panic. Behaviour Research and Therapy, 24, 461-470.

Clark, D., \& Salkowskis, P. (2009). Panic disorder. Manual for Improving Access to Psychological Therapies (IAPT) High Intensity CBT Therapists. https://oxcadatresources.com/wp-content/uploads/2018/06/Cognitive-Therapy-for-PanicDisorder_IAPT-Manual.pdf

Craske, M. G. (2014). Maximizing exposure therapy: an inhibitory learning approach. Behaviour Research and Therapy, 58, 10-23. https://doi.org/10.1016/j.brat.2014.04.006

Craske, M. G., \& Barlow, D. H. (2014). Panic disorder and agoraphobia. In Barlow, D. H. (ed), Clinical Handbook of Psychological Disorders: A Step-by-Step Treatment Manual (pp. 1-61). Guilford Press.

Craske, M. G., \& Simos, G. (2013). Panic disorder and agoraphobia. In Simos, G., \& Hofmann, S. (eds), CBT for Anxiety Disorders: A Practitioner Book (pp. 3-24). Wiley-Blackwell.

De Brey, H. (1983). A cross-national validation of the Client Satisfaction Questionnaire: the Dutch experience. Evaluation and Program Planning, 6, 395-400.

Deacon, B., Lickel, J., \& Abramowitz, J. S. (2008). Medical utilization across the anxiety disorders. Journal of Anxiety Disorders, 22, 344-350. https://doi.org/10.1016/j.janxdis.2007.03.004

Emmelkamp, P. M. G. (2005). Technological innovations in clinical assessment and psychotherapy. Psychotherapy and Psychosomatics, 74, 336-343. https://doi.org/10.1159/000087780

Francis, J. L., Weisberg, R. B., Dyck, I. R., Culpepper, L., Smith, K., Orlando Edelen, M., \& Keller, M. B. (2007). Characteristics and course of panic disorder and panic disorder with agoraphobia in primary care patients. Primary Care Companion Journal of Clinical Psychiatry, 9, 173-179. https://doi.org/10.4088/pcc.v09n0301

Furukawa, T. A., Katherine Shear, M., Barlow, D. H., Gorman, J. M., Woods, S. W., Money, R., Etschel, E., Engel, R. R., \& Leucht, S. (2009). Evidence-based guidelines for interpretation of the Panic Disorder Severity Scale. Depression and Anxiety, 26, 922-929. https://doi.org/10.1002/da.20532

Garcia-Palacios, A., Botella, C., Hoffman, H., \& Fabregat, S. (2007). Comparing acceptance and refusal rates of virtual reality exposure vs. in vivo exposure by patients with specific phobias. CyberPsychology and Behavior, 10, $722-724$. https://doi.org/10.1089/cpb.2007.9962

Houck, P. R., Spiegel, D. A., Shear, M. K., \& Rucci, P. (2002). Reliability of the self-report version of the Panic Disorder Severity Scale. Depression and Anxiety, 15, 183-185. https://doi.org/10.1002/da.10049

Kessler, R. C., Chiu, W. T., Jin, R., Ruscio, A. M., Shear, K., \& Walters, E. E. (2006). The epidemiology of panic attacks, panic disorder, and agoraphobia in the National Comorbidity Survey Replication. Archives of General Psychiatry, 63, 415-424. https://doi.org/10.1001/archpsyc.63.4.415

Kroenke, K., Spitzer, R. L., \& Williams, J. B. W. (2001). The PHQ-9: validity of a brief depression severity measure. Journal of General Internal Medicine, 16, 606-613.

Lindner, P., Miloff, A., Hamilton, W., Reuterskiöld, L., Andersson, G., Powers, M. B., \& Carlbring, P. (2017). Creating state of the art, next-generation virtual reality exposure therapies for anxiety disorders using consumer hardware platforms: design considerations and future directions. Cognitive Behaviour Therapy, 46, 404-420. https://doi.org/10.1080/16506073. 2017.1280843

Malbos, E., Rapee, R. M., \& Kavakli, M. (2013). A controlled study of agoraphobia and the independent effect of virtual reality exposure therapy. Australian and New Zealand Journal of Psychiatry, 47, 160-168. https://doi.org/10.1177/ 0004867412453626

Marcks, B. A., Weisberg, R. B., \& Keller, M. B. (2009). Psychiatric treatment received by primary care patients with panic disorder with and without agoraphobia. Psychiatric Services: A Journal of the American Psychiatric Association, 60, 823-830. https://doi.org/10.1176/ps.2009.60.6.823

Meyerbroeker, K., Morina, N., Kerkhof, G. A., \& Emmelkamp, P. M. G. (2013). Virtual reality exposure therapy does not provide any additional value in agoraphobic patients: a randomized controlled trial. Psychotherapy and Psychosomatics, 82 , 170-176. https://doi.org/10.1159/000342715

Mitte, K. (2005). A meta-analysis of the efficacy of psycho- and pharmacotherapy in panic disorder with and without agoraphobia. Journal of Affective Disorders, 88, 27-45. https://doi.org/10.1016/j.jad.2005.05.003

NHS England (2018). Improving Access to Psychological Therapy Manual. London, UK: NHS England.

Norton, P. J., \& Price, E. C. (2007). A meta-analytic review of adult cognitive-behavioral treatment outcome across the anxiety disorders. Journal of Nervous and Mental Disease, 195, 521-531. https://doi.org/10.1097/01.nmd.0000253843. 70149.9a

Opriş, D., Pintea, S., García-Palacios, A., Botella, C., Szamosközi, Ş., \& David, D. (2012). Virtual reality exposure therapy in anxiety disorders: a quantitative meta-analysis. Depression and Anxiety, 29, 85-93. https://doi.org/10.1002/da.20910

Öst, L.-G. (2008). Cognitive behavior therapy for anxiety disorders: 40 years of progress. Nordic Journal of Psychiatry, 62 (suppl 47), 5-10. https://doi.org/10.1080/08039480802315590

Pelissolo, A., Zaoui, M., Aguayo, G., Yao, S. N., Roche, S., Ecochard, R., Gueyffier, F., Pull, C., Berthoz, A., Jouvent, R., \& Cottraux, J. (2012). Virtual reality exposure therapy versus cognitive behavior therapy for panic disorder with agoraphobia: a randomized comparison study. Journal of Cyber Therapy \& Rehabilitation, 5, 34-43. 
Pompoli, A., Furukawa, T. A., Imai, H., Tajika, A., Efthimiou, O., \& Salanti, G. (2016). Psychological therapies for panic disorder with or without agoraphobia in adults: a network meta-analysis. Cochrane Library, 4, CD011004. https://doi.org/ 10.1002/14651858.CD011004.pub2

Powers, M. B., \& Emmelkamp, P. M. G. (2008). Virtual reality exposure therapy for anxiety disorders: a meta-analysis. Journal of Anxiety Disorders, 22, 561-569. https://doi.org/10.1016/j.janxdis.2007.04.006

Roy-Byrne, P. P., Stein, M. B., Russo, J., Mercier, E., Thomas, R., McQuaid, J., Katon, W. J., Craske, M. G., Bystritsky, A., \& Sherbourne, C. D. (1999). Panic disorder in the primary care setting: comorbidity, disability, service utilization, and treatment. Journal of Clinical Psychiatry, 60, 492-499; quiz 500. https://doi.org/10.4088/jcp.v60n0713

Ruwaard, J., Broeksteeg, J., Schrieken, B., Emmelkamp, P., \& Lange, A. (2010). Web-based therapist-assisted cognitive behavioral treatment of panic symptoms: a randomized controlled trial with a three-year follow-up. Journal of Anxiety Disorders, 24, 387-396. https://doi.org/10.1016/j.janxdis.2010.01.010

Sánchez-Meca, J., Rosa-Alcázar, A. I., Marín-Martínez, F., \& Gómez-Conesa, A. (2010). Psychological treatment of panic disorder with or without agoraphobia: a meta-analysis. Clinical Psychology Review, 30, 37-50. https://doi.org/10.1016/j.cpr. 2009.08.011

Sheehan, D. V., Lecrubier, Y., Sheehan, K. H., Amorim, P., Janavs, J., Weiller, E., Hergueta, T., Baker, R., \& Dunbar, G. C. (1998). The Mini-International Neuropsychiatric Interview (M.I.N.I.): the development and validation of a structured diagnostic psychiatric interview for DSM-IV and ICD-10. The journal of clinical psychiatry, 59 (Suppl 20), 22-33;quiz 34.

Sullivan, B. O., Alam, F., \& Matava, C. (2018). Creating low-cost 360-degree virtual reality videos for hospital. A Technical Paper on the Do's and Don'ts. https://doi.org/10.2196/jmir.9596

Svensson, M., Nilsson, T., Johansson, H., Viborg, G., Perrin, S., \& Sandell, R. (2019). Psychometric analysis of the Swedish panic disorder severity scale and its self-report version. Nordic Journal of Psychiatry, 73, 58-63. https://doi.org/10.1080/ 08039488.2018.1554699

The WHOQOL Group (1995). The World Health Organization Quality of Life assessment (WHOQOL): position paper from the World Health Organization. Social Science Medicine, 41, 1403.

Ustün, T. B., Chatterji, S., Kostanjsek, N., Rehm, J., Kennedy, C., Epping-Jordan, J., Saxena, S., von Korff, M., \& Pull, C. (2010). Developing the World Health Organization Disability Assessment Schedule 2.0. Bulletin of the World Health Organization, 88, 815-823. https://doi.org/10.2471/blt.09.067231

Wolitzky-Taylor, K., Zimmermann, M., Arch, J. J., De Guzman, E., \& Lagomasino, I. (2015). Has evidence-based psychosocial treatment for anxiety disorders permeated usual care in community mental health settings? Behaviour Research and Therapy, 72, 9-17. https://doi.org/10.1016/j.brat.2015.06.010

Cite this article: Lundin J, Lundström A, Gulliksen J, Blendulf J, Ejeby K, Nyman H, Björkander D, and Hedman-Lagerlöf E (2022). Using 360-degree videos for virtual reality exposure in CBT for panic disorder with agoraphobia: a feasibility study. Behavioural and Cognitive Psychotherapy 50, 158-170. https://doi.org/10.1017/S1352465821000473 